\title{
Selection of VolP CODECs for Different Networks based on QoS Analysis
}

\author{
Malik Ahsan Ali \\ Electrical Engineering \\ Department \\ MCS, National University of \\ Science and Technology, \\ Islamabad, Pakistan.
}

\author{
Imran Rashid \\ Electrical Engineering \\ Department \\ MCS, National University of \\ Science and Technology, \\ Islamabad, Pakistan.
}

\author{
Adnan Ahmed Khan \\ Electrical Engineering \\ Department \\ MCS, National University of \\ Science and Technology, \\ Islamabad, Pakistan.
}

\begin{abstract}
Voice over Internet Protocol (VoIP) has been an interesting topic of research in the last decade. The engrossing increase in the use of VoIP services is resulting in the enormous growth of broadband network. The main objective of this paper is the selection of an appropriate voice compression and decompression (CODEC) schemes depending on the Quality of Service (QoS) of VoIP in different networks. Wired, Wireless Local Area Network (WLAN), Worldwide Interoperability for Microwave Access (WiMAX) and Universal Mobile Telecommunication System (UMTS) networks were implemented in OPNET Modeler. The quality is compared using different QoS parameters like end-to-end delay, MOS, throughput and jitter. The VoIP codecs used in the measurements of QoS are: GSM-FR, G.711, G.723.1 and G.729A. Simulations showed that G.711 and GSM- FR are the best schemes that provide high quality of voice in Wireless Local Area Network (WLAN) communications. In WiMAX, G.729A gives the best quality of VoIP while in UMTS, GSM- FR gives overall best results with respect to all the parameters. Wired model gives the best result irrespective of the codec being used. G.723.1 can be used in WiMAX and UMTS along with the wired network depending on conditions. The results analyzed and the performance evaluated will give network operators an opportunity to select the codec for better services of VoIP for customer satisfaction.
\end{abstract}

\section{Keywords}

VoIP; WLAN; WIMAX; UMTS; Codec; QoS.

\section{INTRODUCTION}

Voice over Internet Protocol (VoIP) practices is potentially mounting day by day resulting in the demand of rapid improvements in the networks. There is a demand of decreasing the difference between the qualities of voice and increasing the available bandwidth to provide the best VoIP services comparative to the traditional circuit switched telephony [1]. VoIP has almost replaced the conventional Public Switched Telephone Network (PSTN) due to its cost effectiveness and the features being provided [2]. The wired Internet Protocol (IP) networks provide better VoIP services as compared to the wireless IP network as wireless networks have their own characteristics and impairments [3]. The unsolved issues caused by the wireless network in this area still needs some dedicated work spotlighting VoIP calls. In next generation networks wired and wireless systems have been combined in an innovative way under a single framework [5]. The frequent handovers cause delay and packet loss in these network [6]. The VoIP call gets degraded and loses the packets more swiftly. An eternal solution is required for these heterogeneous systems for the VoIP communication.
The major benefits that wireless network provide is the mobility of the users. It becomes even more challenging on combining the wireless network with multimedia and realtime services to provide QoS [7]. In wireless communication fading influences the channel and degrades the quality of VoIP [8]. WiMAX network has remained the main area of interest for the researchers for VoIP analysis and other networks have rarely been studied [9]-[12]. A correlation has been concluded between the codec being used, amount of voice frames and the delay [13]. The effect of delay on VoIP calls over the WiMAX network depends on the distance and number of base stations [14]. The performance of G.723.1 VoIP CODEC and measurement of the capacity of a WiMAX test bed for up-link and down-link was studied in [15]. Mean Optimum Score (MOS) is the main parameter on which the quality of voice can be concluded. The performance of network using Session Initiation Protocol (SIP) is compared in IPv4 and IPv6 networks [16]. The Windows Operating systems can use either IPv4 or IPv6 and to obtain best VoIP performance the preferred codec can be selected [17].

The researchers have been able to provide some good results but as most of the IP networks and their underlying protocols [4] in use today were implemented keeping in mind the data services not the real-time and delay sensitive voice services [18], there is still a need of providing better QoS as per the demand of the users. A variety of CODECs are being used today each having its own characteristics [19]. Mostly used codec for VoIP is G.711 which provide good results for wired network depending on the environment and conditions [20] but when it comes to wireless networks the quality is degraded. The internet services are becoming so complex that VoIP performance parameters require some actual measurements unlike the traditional telephone networks which were dependent on mathematical modeling. This research is to provide good quality of VoIP services in every network and analysis is done using different codecs mentioned in table1. VoIP packets are analyzed focusing all the major parameters like end-to-end delay, MOS, throughput and jitter over Wired, Wireless, UMTS and WiMAX networks using the OPNET Modeler..

\section{VOIP AND CODECS}

The demand for mobile and broadband services is rising day by day. The last decade has seen the ever-increasing VoIP users with the demand of reliable and quality services. VoIP is an emerging technology for voice communication used these days. The services are not only being used for long distance calls but also for the short distant communications. The devices like IP phones and the VoIP enabled desktop systems are cost effective and also provide some new features to the users. Keeping in mind the demand of the users, the operators are forced to improve the quality of communication. 
This can be achieved by increasing the bandwidth and making the IP backhaul that fulfills the demand of the users at lower cost providing better QoS.

\subsection{VoIP Codecs}

Codec is a coder/decoder which converts the audio signal to digitized version for transmission over the medium and then back into the original uncompressed version on the receiver side. This concept is the base of VoIP services. There are a number of codec used for VoIP communication each having its own bandwidth and characteristics. The codecs which are used in this research work are listed in the table I below.

Table 1. Characteristics of VoIP Codecs

\begin{tabular}{|c|c|c|}
\hline CODEC & Coding Algo & Sampling rate \\
\hline GSM- FR & PRE-LTP & $13 \mathrm{kbps}$ \\
\hline G.711 & PCM & $64 \mathrm{kbps}$ \\
\hline G.723.1 & ACELP & $5.3 \mathrm{kbps}$ \\
\hline G.729A & CS-ACELP & $8 \mathrm{kbps}$ \\
\hline
\end{tabular}

\section{NETWORK MODELS}

The tool used for simulations is OPNET Modeler as it provides the results very closer to the real time environment. The models were created by selecting the nodes and links from the object palette such that to reduce the losses/impairments effect. Wired model designed, is a general IP network. Links in the wired design as shown in figure 1 consist of standard 100baseT lines from user to router and from router to internet cloud followed internet server is $\mathrm{T} 1$ line. WLAN design consists of user node and access point connected to the IP backhaul with a T1 line as shown in figure 2. UMTS model as in figure 3 comprises user equipments, node B and Radio Network Controller (RNC) which is connected to the packet switched network via Serving GPRS Support Node (SGSN) and Gateway GPRS Support Node (GGSN) which in turn is connected to the IP Network. Figure 4 represents the WiMAX model which is designed using the base station connected to the IP backhaul serving the VoIP users. A T1 line is used to simulate a perfect connection between router and server minimizing cable delay and allowing the difference caused by the codecs to be more noticeable. The attributes and parameter settings are made in the network models and various simulations are carried out for the codecs. The reason for utilizing this modeling method is to allow performance of the codecs to be analyzed in an improved manner.

\subsection{Wired}

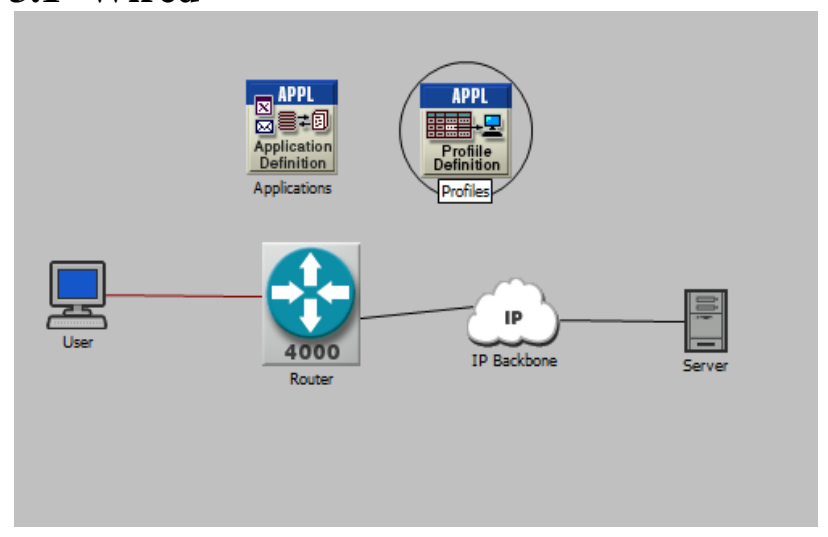

Fig. 1: Wired Model

\subsection{WLAN(wireless)}

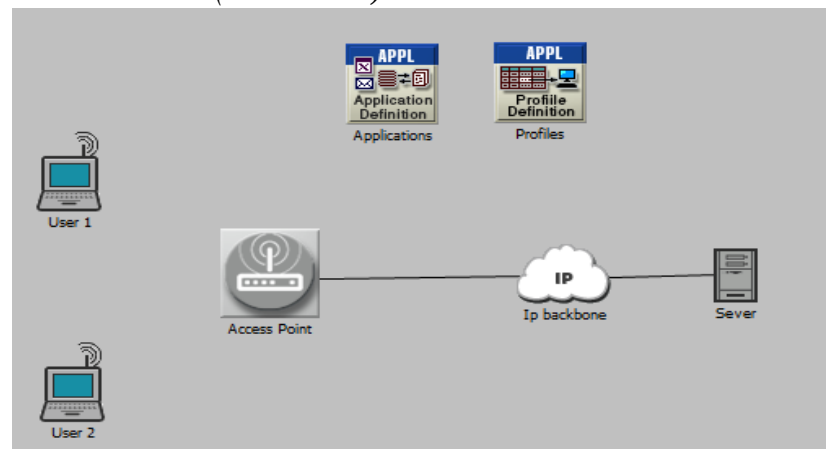

Fig. 2: WLAN Model

\subsection{UMTS}

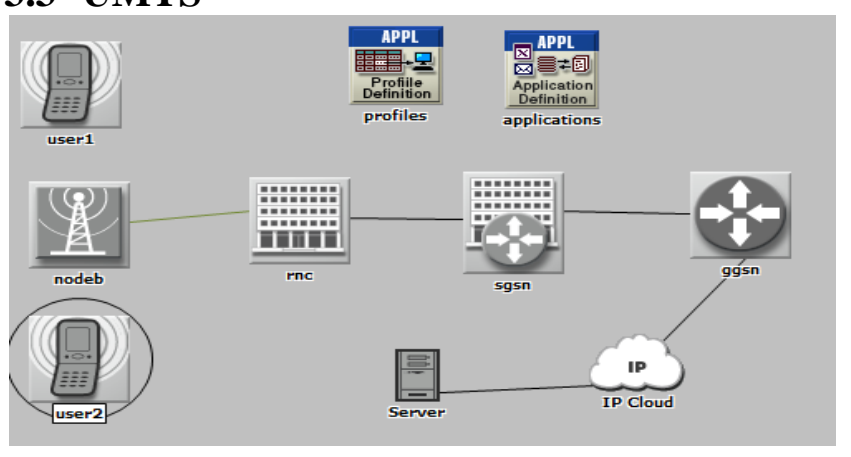

Fig. 3: UMTS Model

\subsection{WiMAX}

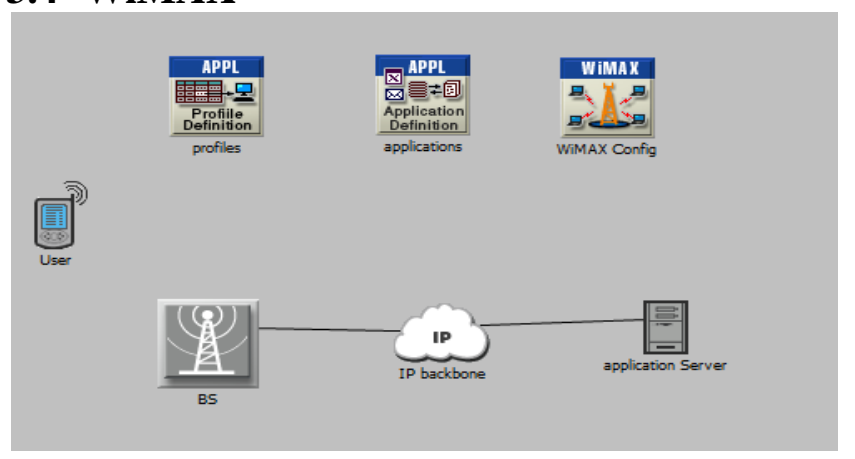

Fig. 4: Wimax Model 


\section{RESULTS AND ANALYSIS}

The comparative analysis of UMTS, WiMAX, Wired and WLAN networks using each codec while keeping the simulation environment and attributes same, is discussed in this section. The performance of each codec is evaluated in the network models depending on the QoS.

\subsection{Analysis of Codec G.711}

This simulation is performed for G.711 codec in different networks. The results shown below are used to evaluate the performance of G.711. It is analyzed from figure 5 that the value of Mean Opinion Score (MOS) is 3.7 in wired, wireless and WiMAX models, showing the good quality of speech. Comparatively, MOS value for UMTS model is 2.5 showing the worst quality of speech amongst all the models. Jitter and end to end delay in figures 6 and 7 shows that UMTS and WiMAX models undergo a delay in packets and attain some jitter. In UMTS a jitter and significant amount of delay is attained degrading the quality while in WiMAX, delay and packet loss effect the communication. Traffic sent is almost same in all the models as shown in figure 8 while the traffic received in figure 9 shows that in WiMAX and UMTS there is loss in the packets as compared to wired and WLAN networks. Jitter, delay and less reception of packets in UMTS model represent that it gives worst quality of voice while using G.711. The performance of WiMAX models is also not effective as there is a delay and it loses packets. Wired and WLAN models give best performance while using G.711.

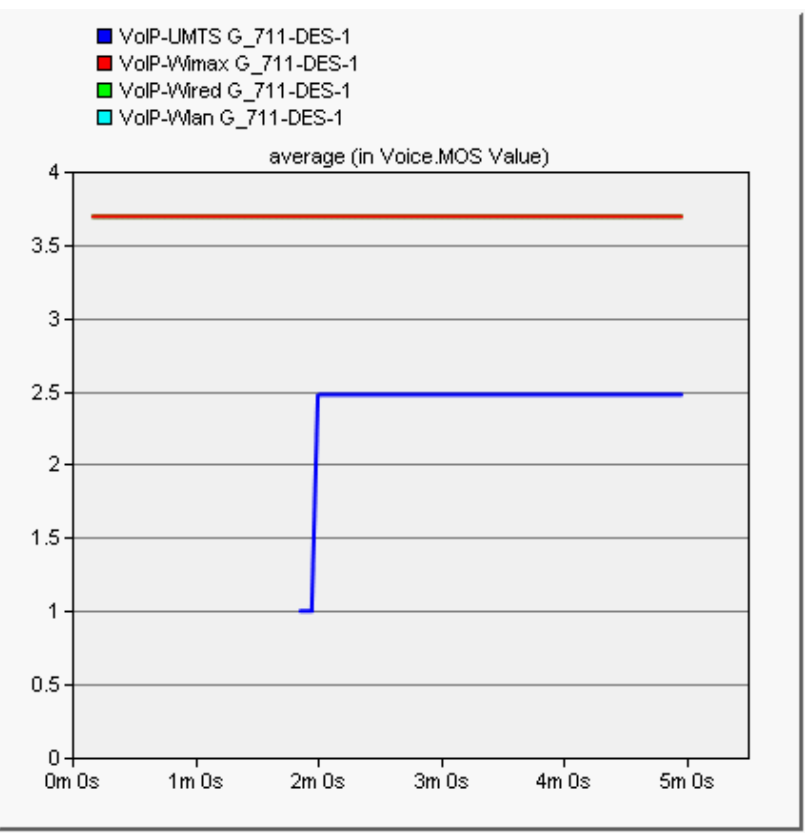

Fig. 5: MOS for G.711

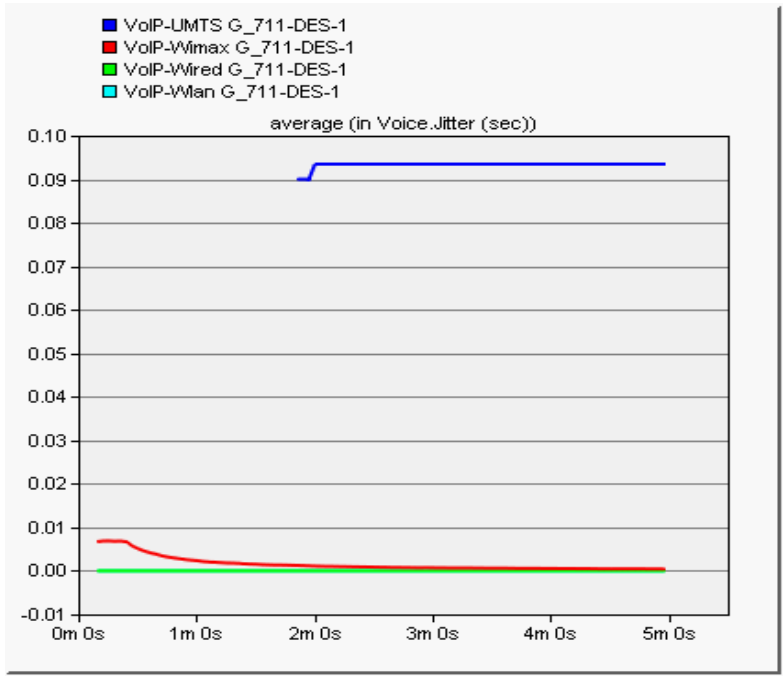

Fig. 6: Jitter for G.711

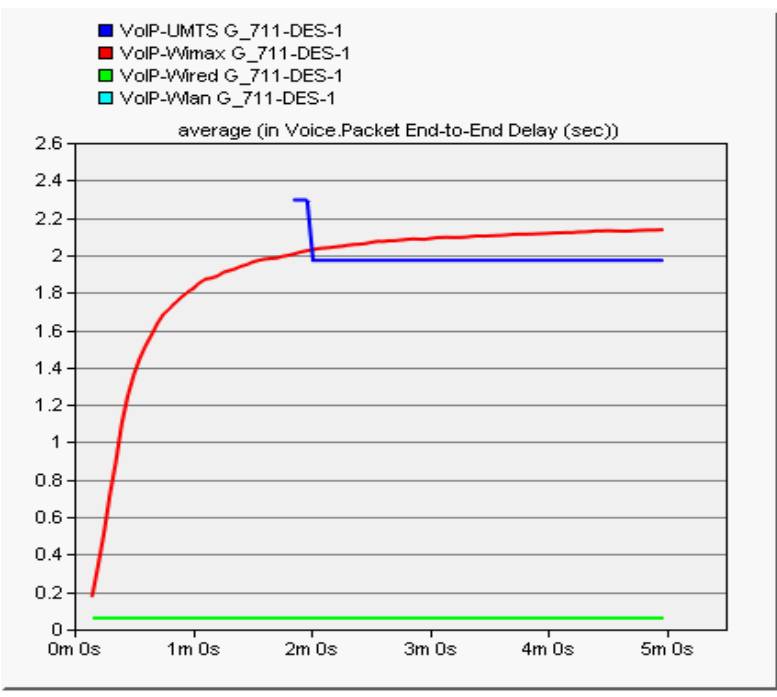

Fig. 7: Packet end to end delay for G.711

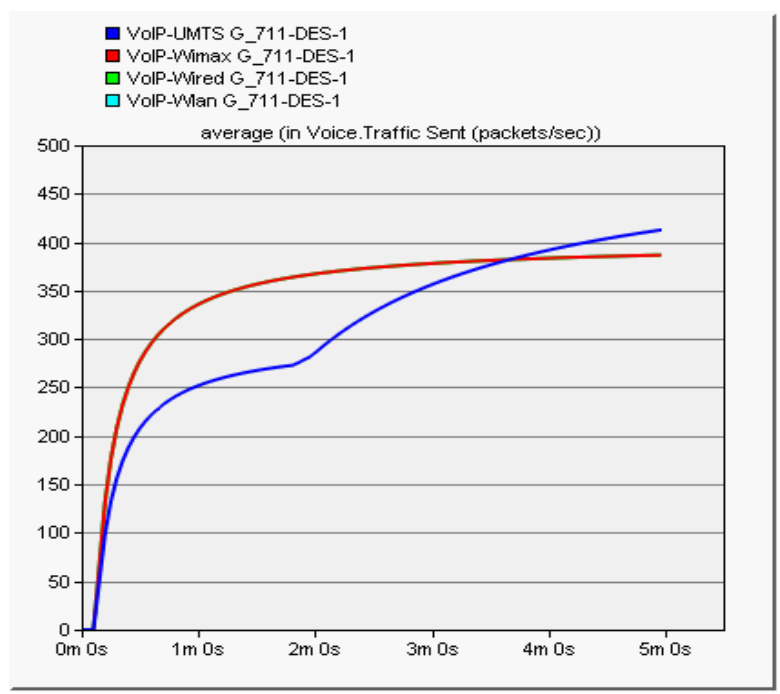

Fig. 8: Traffic sent for G.711 


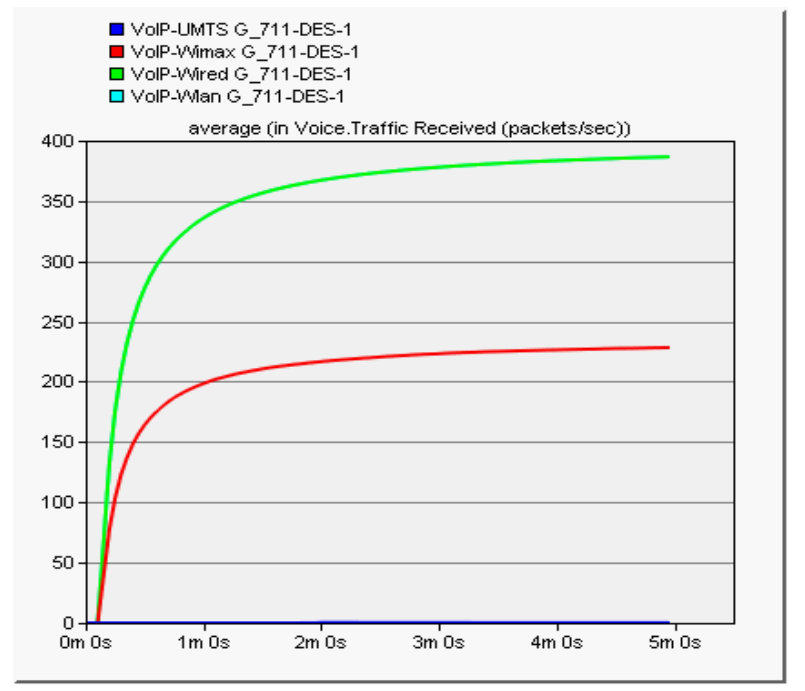

Fig. 9: Traffic Received for G.711

\subsection{Analysis of Codec G.723.1}

This simulation is performed for G.723.1 codec in different networks. The results shown in figure 10-14 are used to evaluate the performance of G.723.1. Figure 10 show that the value of MOS is 2.5 in wired, WLAN and WiMAX models. Comparatively, MOS value for UMTS model is 2 showing the bad quality of speech. However when it comes to jitter and end to end delay in figures 11 and 12, WLAN and UMTS models along with the wired model have minimum delay in packets and attain zero jitter. In WiMAX jitter and the amount of delay is very small hence providing the good quality of VoIP. Traffic sent and received is almost same in all the models except WiMAX model which loses a small amount of packets as shown in figures 13 and 14. Jitter, delay and full reception of packets in wired, WLAN and UMTS models allow using G.723.1 codec with low MOS. The performance of wired, WLAN, WiMAX and UMTS models is not quite effective but the jitter and delay is reduced using G.723.1 codec.

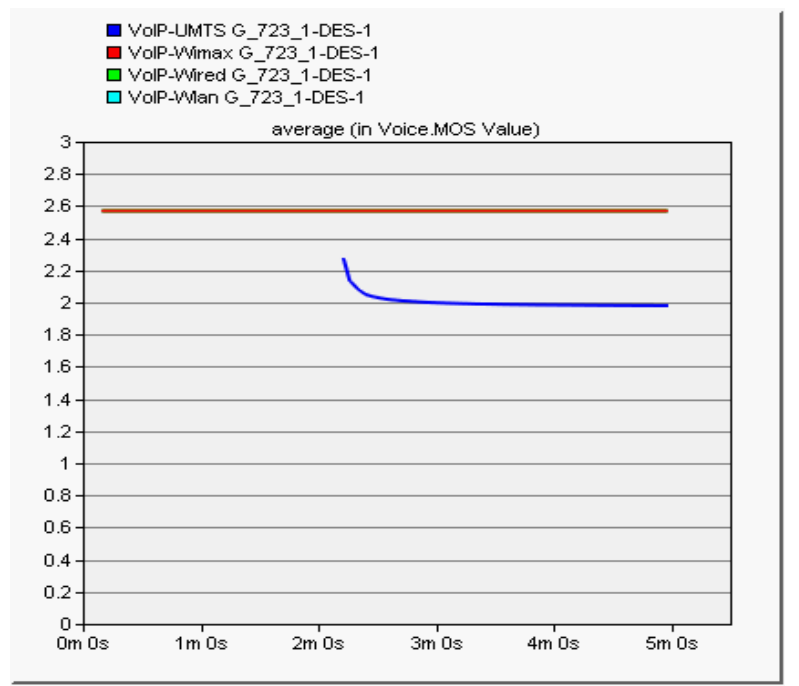

Fig. 10: MOS for G.723.1

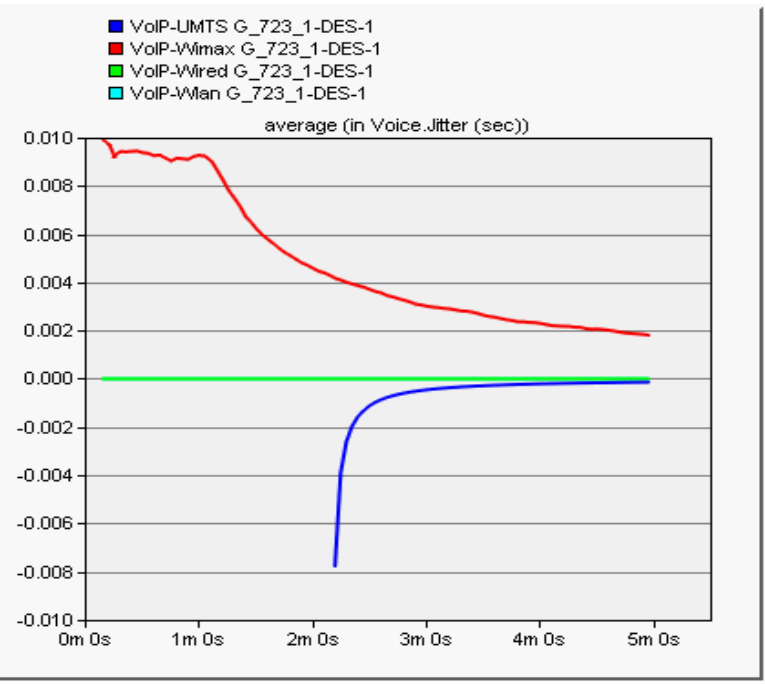

Fig. 11： Jitter for G.723.1

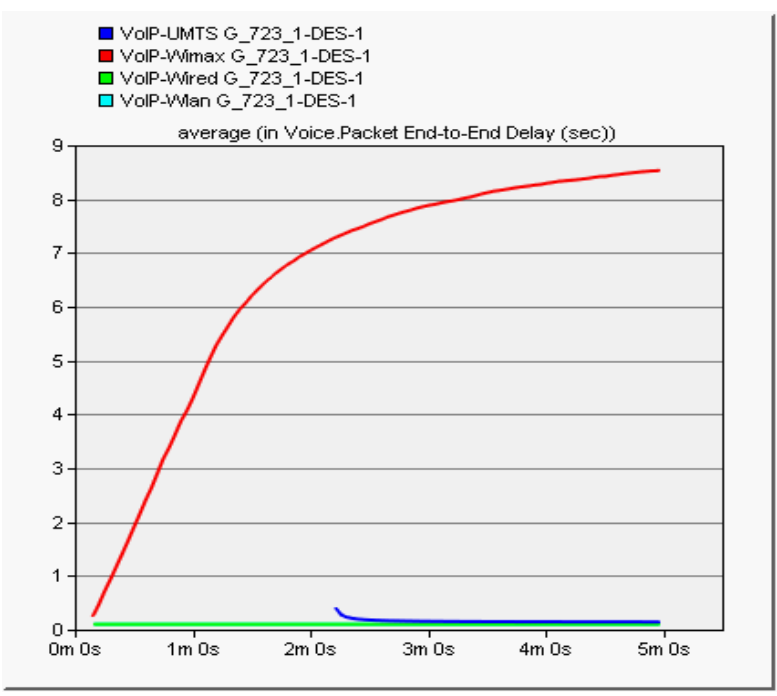

Fig. 12: Packet end to end delay for G.723.1

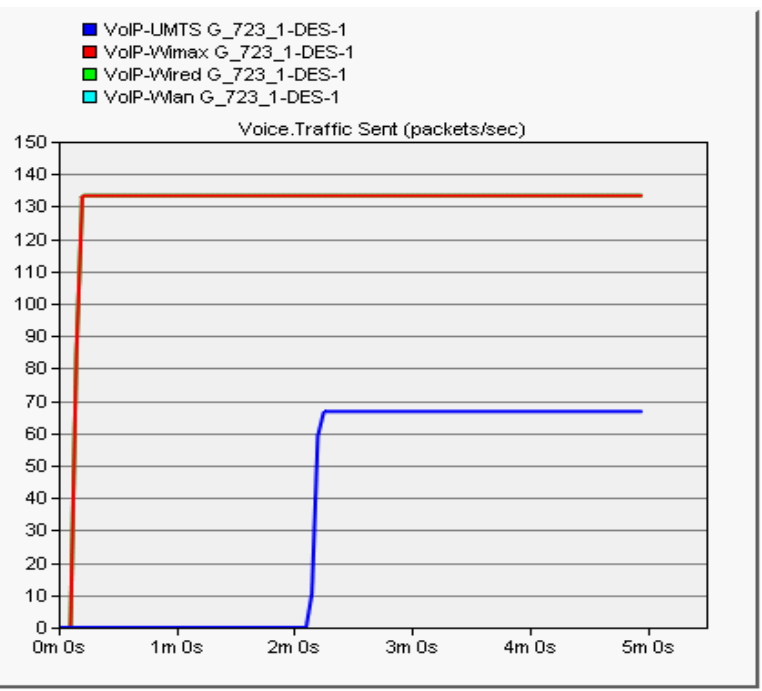

Fig. 13: Traffic sent for G.723.1 


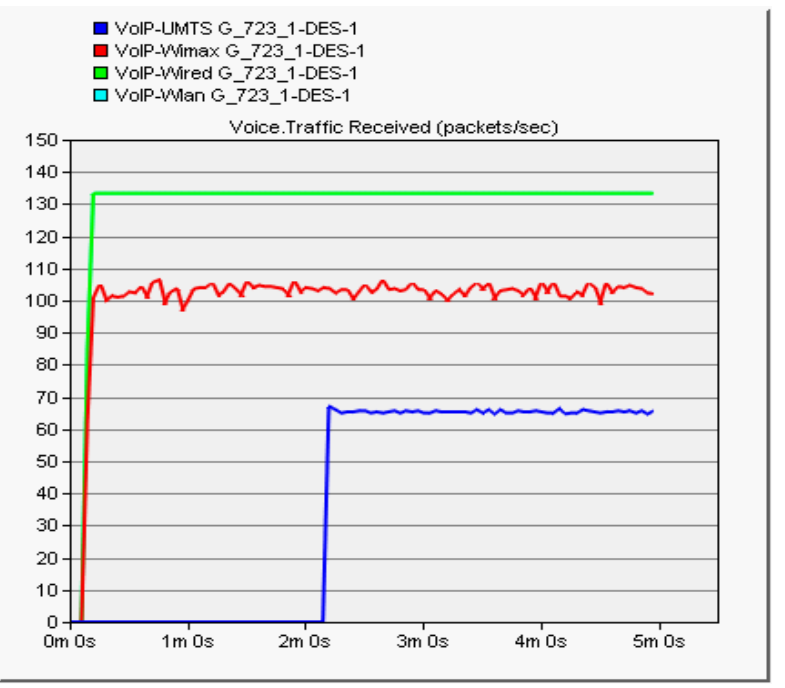

Fig. 14: Traffic Received for G.723.1

\subsection{Analysis of Codec G.729A}

This simulation is performed for G.729A codec in different networks. The results shown in figure 15-19 are used to evaluate the performance of G.729A. Figure 15 show that the value of MOS is 3.2 in wired, WLAN and WimAX models. Comparatively, MOS value for UMTS model is 2.4 showing the bad quality of speech. WiMAX model along with the wired model show the best quality of VoIP. Jitter and end to end delay in figures 16 and 17 shows that WLAN and UMTS models undergo a delay in packets and attain some jitter which in turn loses the packets. In WiMAX jitter and the amount of delay is very small hence providing the good quality of VoIP. Traffic sent and received is almost same in wired and WiMAX models while there is some loss of packets in WLAN and UMTS networks as shown in figure 23 and 24. Jitter, delay and full reception of packets in WiMAX model represent that it gives best quality of voice while using G.729A. The performance of WLAN and UMTS models is not effective as there is a delay and packets are lost.

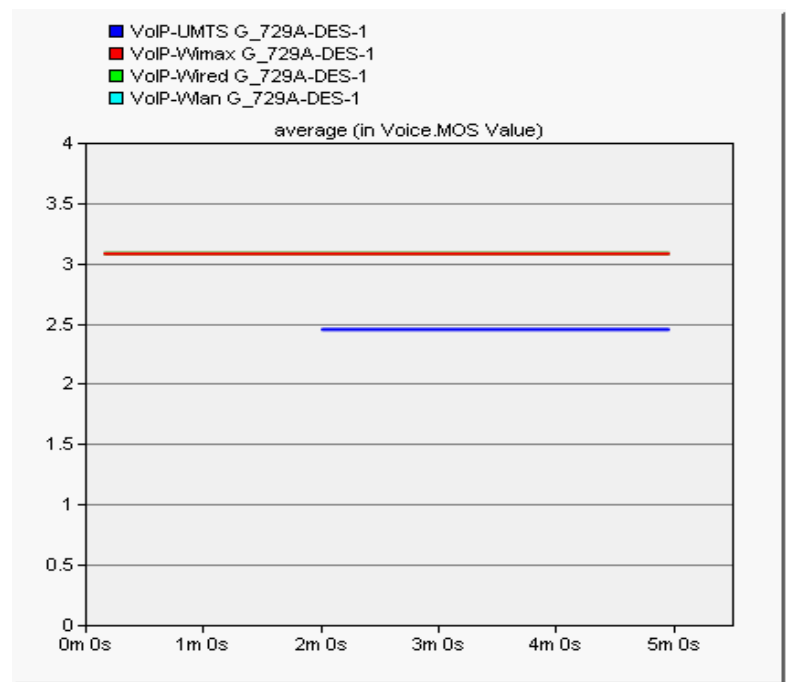

Fig. 15: MOS for G.729A

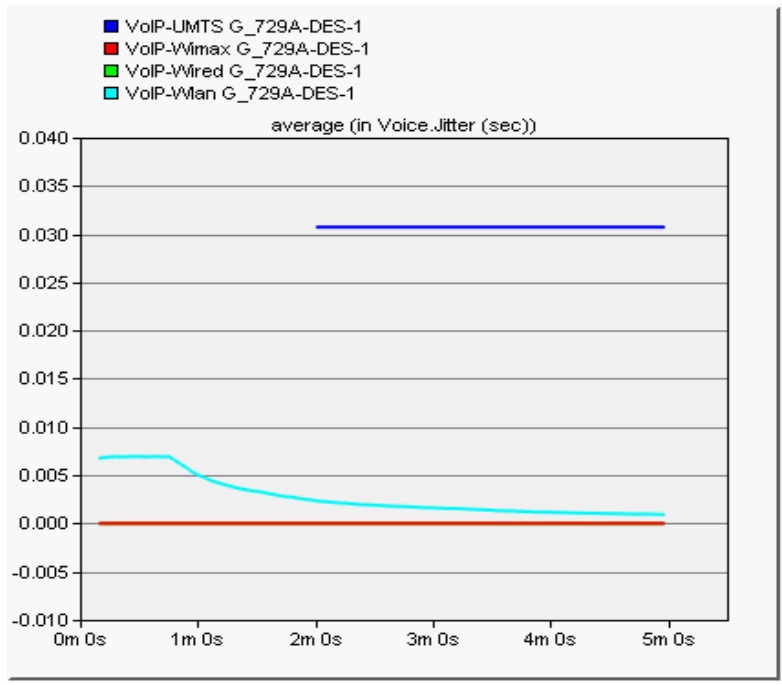

Fig. 16: Jitter for.729A

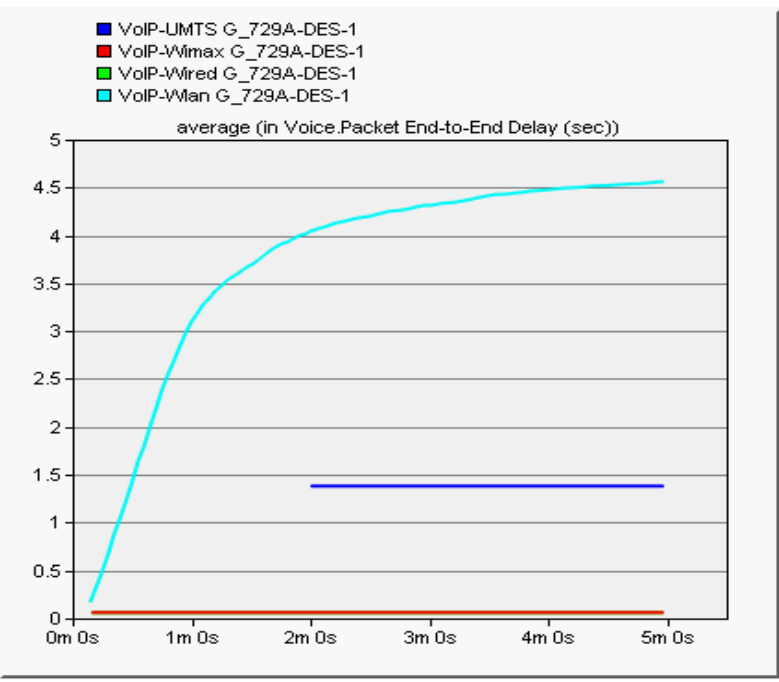

Fig. 17: Packet end to end delay for G.729A

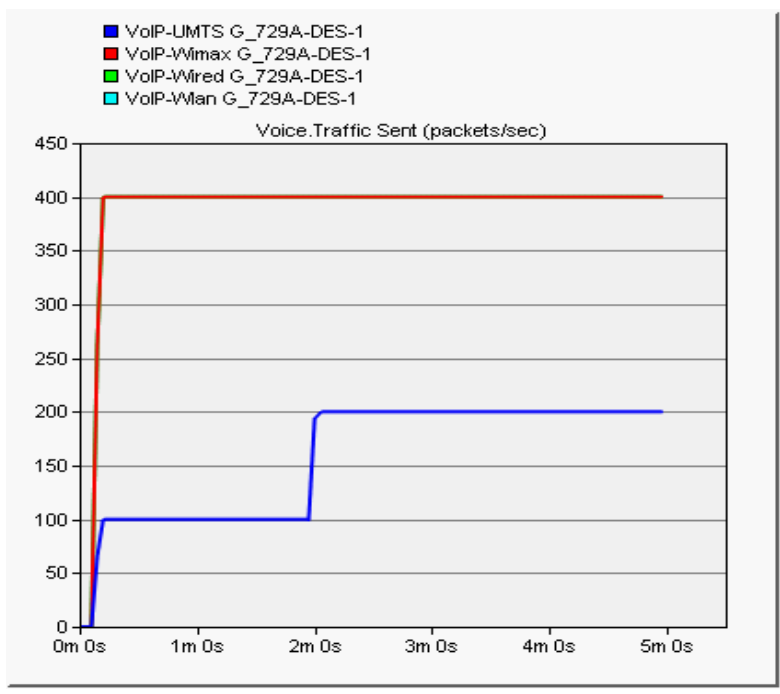

Fig. 18: Traffic sent for G.729A 


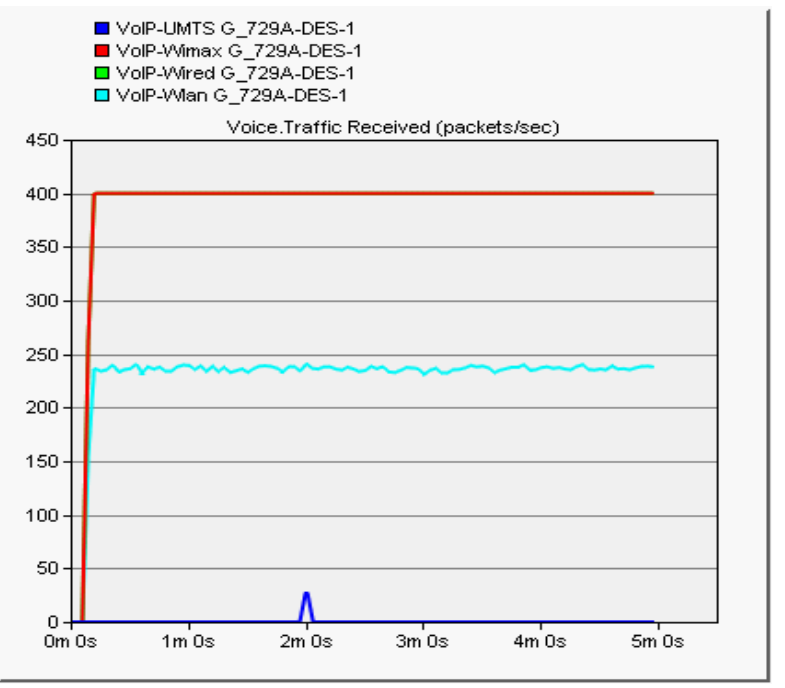

Fig. 19: Traffic Received in G.729A

\subsection{Analysis of Codec GSM-FR}

This simulation is performed for GSM-FR codec in different networks. The results shown below in figure 20-24 are used to evaluate the performance of GSM- FR. Figure 20 shows that the value of MOS is 3.6 in wired and WLAN models. Comparatively, MOS value for UMTS model is 3 showing the good quality of speech. WiMAX model shows the worst quality amongst all the networks. Jitter and end to end delay in figures 21 and 22 shows that WiMAX model undergoes a delay in packets and attain some jitter which in turn loses the packets. In UMTS jitter and the amount of delay is very small hence providing the good quality of VoIP. Traffic sent and received is almost same in all the models except WiMAX as shown in figure 23 and 24. Jitter, delay and less reception of packets in WiMAX model represent that it gives worst quality of voice while using GSM-FR. The performance of UMTS model is very much effective as there is no delay and it does not lose packets. Wired and WLAN models give the best performance.

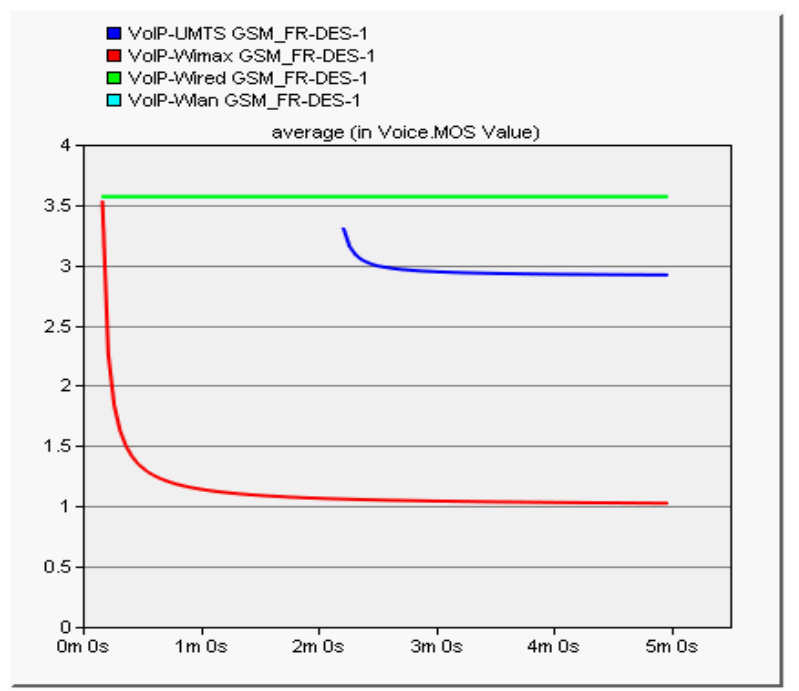

Fig. 20: MOS for GSM-FR

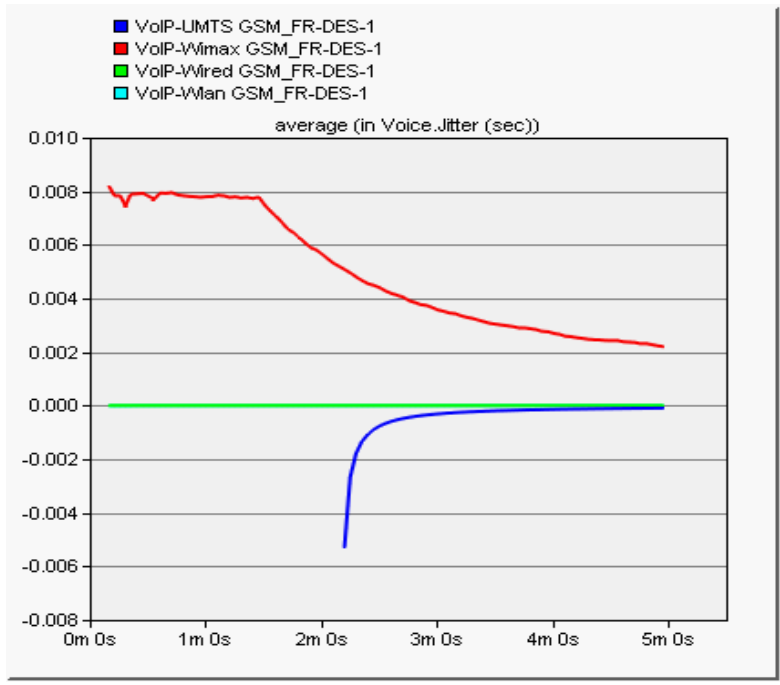

Fig. 21： Jitter for GSM-FR

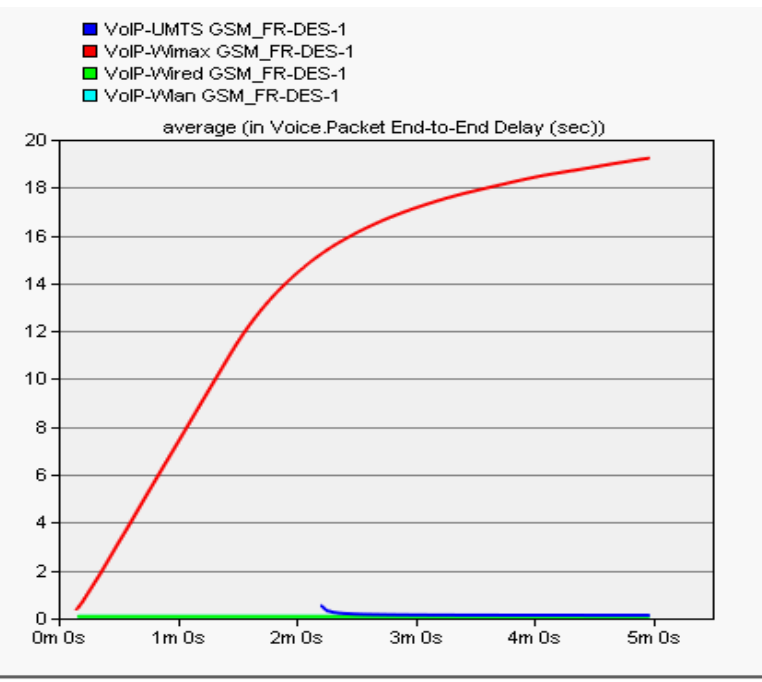

Fig. 22: Packet end to end delay for GSM-FR

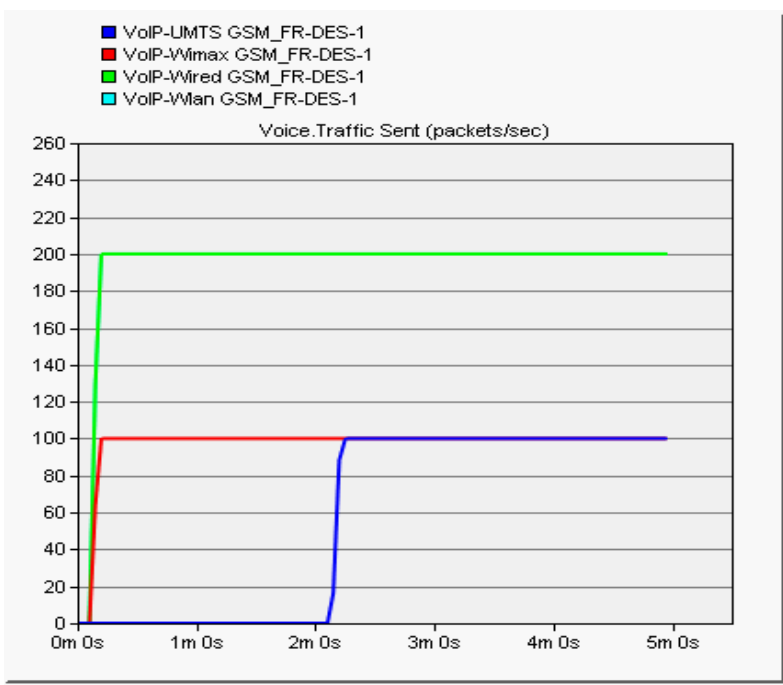

Fig. 23: Traffic sent for GSM-FR 


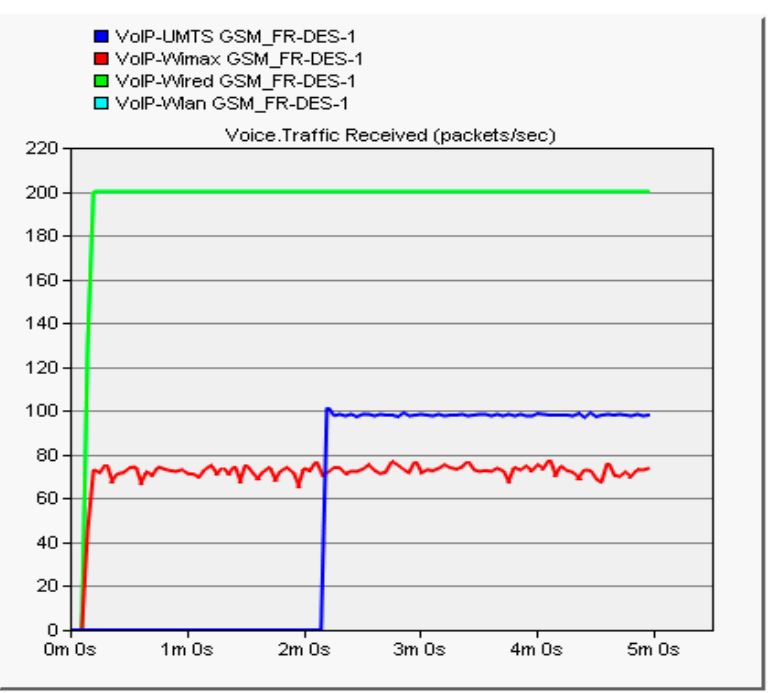

Fig. 24: Traffic Received for GSM-FR

\section{CONCLUSION AND FUTURE WORK}

Performance of various VoIP codecs in different networks is analyzed using the OPNET Modeler. A variety of simulations are carried out to get the most effective and efficient results. On the basis of results attained, conclusion for the selection of VoIP codecs in different networks is made. Depending on the results it is concluded that wired network performs well irrespective of the VoIP codec being used. G.711 and GSMFR can be selected for VoIP communications in WLAN network. For WiMAX network, G.729A codec is the most effective one. In UMTS network the best VoIP quality is given while using GSM-FR. The quality of G.723.1 codec is observed low as it is a low quality codec. Hence it can be used in all the networks depending on the environment and users density. The conclusions will be helpful for the network operators and also for the researchers to further work on the topic. The mobility parameter of the wireless models will be the main focus of the future work.

\section{REFERENCES}

[1] T. Kwok, "Residential broadband Internet services and applications requirements" Communications Magazine, IEEE Volume 35, Issue 6, June 1997 Page(s):76 - 83

[2] T. Wallingford, "Switching to VoIP", Publisher: O'Reilly, ISBN: 0-596-00868-6, Pub Date: June 2005

[3] A. Samukic, "UMTS Universal Mobile Telecommunications System Development of Standards for the Third Generation", 1998

[4] IEEE Std 802.11, "Wireless LAN Medium Access Control(MAC) and Physical Layer (PHY) Specifications," ANSI/IEEE Std 802.11, 1999.

[5] Per Beming, Lars Frid, Göran Hall, Peter Malm, Thomas Noren, Magnus Olsson and Göran Rune, "LTE-SAE architecture and performance," Ericsson Review No. 3, 2007

[6] Naveed Iqbal, Ajmal Khan, Malik Ahsan Ali, Uzma Anwar, Burhan Ullah, M. Faizan Sabir, " Performance Analysis of Soft and Hard Handovers based on UMTS QoS Traffic Classes," ICIIT, 2010.
[7] Muhammad Imran Tariq, Muhammad Ajmal Azadand Syed Khurram Rizvi. " Effect of Mobility Patterns on VoIP QoS in Mobile WiMAX," International Journal of Computer Science and Telecommunications,vol. 4, 2013

[8] H. Arora and H. Sethu, "A simulation study of the impact of mobility on performance in mobile ad hoc networks," in Proceedings of the Applied Telecommunication Symposium San Diego, California, April 14-18, 2002.

[9] A. A. Ali, S. Vassilaras and K. Ntagkounakis, "A Comparative Study of Bandwidth Requirements of VoIP Codecs over WiMAX Access Networks," Third International Conference on Next Generation Mobile Applications, Services and Technologies (NGMAST '09), 2009, pp. 197 - 203.

[10] K. A. Shuaib, "A Performance Evaluation Study of WiMAX Using Qualnet," Proceedings of The World Congress on Engineering 2009, pp. 912-916.

[11] S. Jadhav, Z. Haibo and H. Zhiyi, "PerformanceEvaluation of Quality of VoIP in WiMAX and UMTS," 12th International Conference on Parallel and Distributed Computing, Applications and Technologies (PDCAT), 2011, pp. 375-380.

[12] M. I. Tariq, M. A. Azad, R. Beuran and Y. Shinoda, "Performance Analysis of VoIP Codecs over BE WiMAX Network," 3rd International Conference on Computer and Electrical Engineering (ICCEE), 2010, vol. 9 , pp. 47-51.

[13] C. Jianguo and M. Gregory,“ Performance Evaluation of VoIP Services using Different CODECs over a UMTS Network," Australasian Telecommunication Networks and Applications Conference (ATNAC), 2008, pp. 6771.

[14] Safak and B. Preveze, "Analysis of delay factors for voice over WiMAX," in Computer and Information Sciences, 2008. ISCIS '08. 23rd International Symposium on, oct. 2008, pp. 1-6.

[15] K. Pentikousis, E. Piri, J. Pinola, F. Fitzek, T. Nissila," and I. Harjula, "Empirical evaluation of voip aggregation over a fixed WiMAX testbed," in Proceedings of the 4th International Conference on Testbeds and research infrastructures for the development of networks \& communities, ser. TridentCom'08, 2008, pp. 19:1-19:10.

[16] T. Hoeher, M. Petraschek, S. Tomic, and M. Hirschbichler. "Evaluating Performance Characteristics of SIP over IPv6," IEEE Journal of Networks,vol. 2, no. 4, pp. 10, 2007.

[17] Hira Sathu and Mohib A. Shah. "Performance Comparison of VoIP Codecs on Multiple Operating Systems using IPv4 and IPv6" International Journal of eEducation, Vol. 2, No. 2, April 2012

[18] R. Dominach "Quality of service for real-time IP applications", TMCnet. March 2004.

[19] ITU-T P.830, "Subjective performance assessment of telephone-band and wideband digital codecs," 1996.

[20] Aamir, M.; Zaidi, S.M.A., "QoS analysis of VoIP traffic for different codecs and frame counts per packet in multimedia environment using OPNET," INMIC, 2012 15th International, vol.,no., pp.275,281, 13-15Dec. 